\title{
Commentary
}

\section{Freundlich Isotherm: An Adsorption Model Complete Framework}

\author{
Michael Vigdorowitsch ${ }^{1,2,3, *}$, Alexander Pchelintsev ${ }^{3} \oplus$, Liudmila Tsygankova ${ }^{4}$ and Elena Tanygina 4 \\ 1 Angara GmbH, Mörsenbroicher Weg 191, 40476 Düsseldorf, Germany \\ 2 All-Russian Scientific Research Institute for the Use of Machinery and Oil Products in Agriculture, \\ Novo-Rubezhny Sidestr. 28, 392022 Tambov, Russia \\ 3 Department of Higher Mathematics, Tambov State Technical University, Sovetskaja Str. 106, \\ 392000 Tambov, Russia; pchelintsev@mail.tstu.ru \\ 4 The G.R. Derzhavin Tambov State University, Internatsionalnaja Str. 33, 392000 Tambov, Russia; \\ vits21@mail.ru (L.T.); tanhe@mail.ru (E.T.) \\ * Correspondence: mv016@yahoo.com or dr.vigdorowitsch@angara-gmbh.de
}

Citation: Vigdorowitsch, M.;

Pchelintsev, A.; Tsygankova, L.;

Tanygina, E. Freundlich Isotherm: An Adsorption Model Complete

Framework. Appl. Sci. 2021, 11, 8078.

https://doi.org/10.3390/

app11178078

Academic Editor: Stefano Salvestrini

Received: 27 May 2021

Accepted: 24 July 2021

Published: 31 August 2021

Publisher's Note: MDPI stays neutral with regard to jurisdictional claims in published maps and institutional affiliations.

Copyright: (C) 2021 by the authors. Licensee MDPI, Basel, Switzerland. This article is an open access article distributed under the terms and conditions of the Creative Commons Attribution (CC BY) license (https:// creativecommons.org/licenses/by/ $4.0 /)$.

\begin{abstract}
The absolute majority of modern studies dealing with the interpretation of experimental data on the basis of the Freundlich isotherm ignore the fact that the data obtained for regions of low and moderate adsorbate concentration/pressure can be analytically continued within the Freundlich adsorption model to the adsorptive saturation area with coverages tending to $100 \%$. Needless to say, this would give valuable extended information about the corresponding adsorption process. This message proposes a framework to comprehensively analyse experimental data first recognised as complying with the Freundlich adsorption model. An algorithm-driven method is presented which enables one to translate the data obtained in the area of small and moderate the coverages of the area of adsorptive saturation regime. As examples, three sets of experimental data for adsorption of mercury (II) on N-rich porous organic polymers and of protein on carrier nano- $\mathrm{Mg}(\mathrm{OH})_{2}$ have been processed and presented according to the framework developed.
\end{abstract}

Keywords: Freundlich isotherm; adsorption; sorption; saturation regime; surface coverage

\section{Introduction}

Long ago the Freundlich isotherm found itself among the isotherms through which one typically checks experimental data obtained. The purpose of this action consists most often of the determination of fitting parameters in order to choose on the $R^{2}$ approximation basis which model out of the de facto standard set (Langmuir-, Temkin-, and maybe, Dubinin-Radushkevich) of models provides the best fit. Should it be the Freundlich model, one could dwell on the degree of energetic inhomogeneity of the surface (the magnitude of parameter $\alpha$ in Equation (1)):

$$
\theta=K x^{\alpha}
$$

where $x$ and $\theta$ are in bulk concentrations (for solutions with a constant ionic power. As $c$, pressure in the gas phase or ionic activity in the solution can be considered) of a surfaceactive substance being adsorbed and related surface coverage, respectively. The power of $x$ is often considered as $\alpha=1 / n$, where $n>0$ is an integer or real number, and the coverage is expressed through adsorption $q$ and limiting adsorption $q_{\infty}$ as $\theta=q / q_{\infty}$. More advanced analysis of fitting results gives rise to a conclusion on which type of the surface (in)homogeneity takes place for a pair "adsorbate/adsorbent" considered, that is, evenly inhomogeneous (Temkin adsorption model, or AM), exponentially inhomogeneous (Freundlich) or homogeneous (Langmuir) (for a more complete set, one could consider a linear inhomogeneity as well [1], but its representation through the proper equation is much more complicated than those for the other three. The Dubinin-Radushkevich AM is to be mentioned again as well but this is more applicable for adsorption on materials with pronounced porous properties). A huge variety of realizations of the Freundlich AM, among 
whose are the works having studied the adsorption of organic and inorganic substances on substrates of different nature in the framework of chemical, physical, biophysical processes including nano-particles as just examples [2-10], seems to represent a substantial reason for revisiting the basics of the Freundlich AM.

While the monograph by McBain first convincingly elucidated the meaning of Equation (1) for interpretation of experimental data [11], the proper theoretical model was developed by Zeldowitch (1934) [12,13], and later reproduced as one of parametrical limits while expanding the general solution for energetically heterogeneous surfaces, expressed through a hypergeometric function into an infinite series [14,15]. The limiting adsorption is bounded through the surface available and an effective number of layers (should it be multi-layer). As it follows from the functional form itself of Equation (1), it does not describe a saturation regime, so that deviations from Equation (1) appear at bigger $x$. It has become typical for the research community, that data obtained and to be analysed in the framework of Freundlich AM, are to be typically checked for their relevance to Equation (1) with a successive determination of constants $K$ and $a$ as fitting parameters. Nevertheless, Zeldowitch's theory of the Freundlich AM goes beyond the small and moderate coverages and mathematically describes the saturation regime too. This circumstance seems to be most typically either ignored or forgotten by the research community although studies with the employment of the Freundlich isotherm for pairs "adsorbate/adsorbent" appear to be published in hundreds (if not more) of articles annually.

On the basis of Zeldowitch's theory, it appears possible to link the area of small coverages and that at saturation. Furthermore, the theory is applicable to both monoor multi-layer adsorption. In the last case, one has to distinguish between successive adsorption of layers "one after one" like a staircase and simultaneous adsorption when successive layers are being filled without completely filling the foregoing ones. For the former, the theory is to be applied to each "stair" separately.

The goal of this message is to revisit Zeldowitch's theory, to propose a mathematical methodology for bridging a chasm between the small (moderate) adsorption regime satisfactorily described by Equation (1) on the one hand and the limiting adsorption on another hand and, thus, cover the whole range of surface coverages within the Freundlich AM. This is to be formulated in the form of an algorithm for the proper analysis of adsorption in those systems where the Freundlich AM is considered the true one. What we need for that is just the experimental data obtained and published by the research community before for small and possibly moderate coverages only.

\section{A Brief Excursus to Zeldowitsch's Theory}

Zeldowitch considered the problem of finding such a distribution $a(b)$ of surface adsorption centres on their adsorption heat that local application of the Langmuir AM would lead to an overall functional form (1) for the whole surface. For any particular surface patch with particular adsorption heat $b$, the Langmuir adsorption is to take place. The patches are arbitrary laterally distributed, $a$ is proportional to the number of patches whereas $b$ depends on their properties $[12,13,16]$. Mathematically, this meant solving the following integral equation

$$
q(x) / q_{\infty}=\int_{0}^{b_{0}} \frac{a(b) x}{x+b} d b
$$

where the upper limit $b_{0}$ was introduced artificially to enforce the integral convergence. Since Equation (2) is intractable with respect to $a(b)$, the problem was simplified through the following replacement: instead of the Langmuir isotherm $\frac{a x}{x+b}$ as the integrand in Equation (2), the following local adsorption function was employed:

$$
\left\{\begin{array}{l}
\frac{a c}{b}, \text { if } x \in(0 ; b] \\
a, \text { if } x \in\left(b ; b_{0}\right]
\end{array}\right.
$$


With such a kernel function in Equation (2), the distribution function $a(b)=A b^{\alpha-1}$ was shown to meet Equation (2), should the isotherm have the form as in Equation (1). The following relationships appear to be an integral part of Zeldowitch's theory: at small $x$

$$
\lim _{x \rightarrow 0} \frac{q(x)}{q_{\infty}}=\left(\frac{x}{b_{0}}\right)^{\alpha} \frac{\pi / n}{\sin \pi / n}
$$

whereas for big $x \gg b_{0}$ one has

$$
\frac{q(x)}{q_{\infty}}=1-\frac{b_{0}}{\left(1+\frac{1}{\alpha}\right)} \frac{1}{x}+\ldots \approx \frac{x}{x+b_{0} /\left(1+\frac{1}{\alpha}\right)}
$$

Thus, should constant $b_{0}$ be approximately determined on the basis of experimental data according to Equation (3) as

$$
b_{0}=\lim _{x \rightarrow 0} c\left(\frac{q_{\infty}}{q(x)} \frac{\pi / n}{\sin \pi / n}\right)^{1 / \alpha}
$$

the saturation regime (4) at big $x$ appears to be identified as well. Noticeable is that, according to Zeldowitch's theory, it has the Langmuir-type functional form but with different adsorption equilibrium constants.

\section{The Consistent Algorithm of Analysis of Experimental Data}

According to the above said, dependencies like Equation (1) appear to be determined and carefully studied on the basis of small and/or moderate concentrations, which include determination of constants $K$ and $\alpha$ (blue curves in Figure 1). The saturation regimes (4) (shown grey in Figure 1) turn out to be, as a rule, omitted both in experimental studies and discussions of obtained results. Nevertheless, there are a lot of evident reasons why such regimes, as well as intermediate regions (orange in Figure 1), can represent considerable interest for the research community and do.

Therefore, in step 1 , upon the determination of the fitting parameters $K$ and $\alpha$, one is expected to determine the saturation regime. It has the Langmuir-like form $f_{3}(x)=\frac{x}{x+b}$ where $b \equiv b_{0} /\left(1+\frac{1}{\alpha}\right)$ (we now redefine constant $b$ as different from that in Equation (2)). Thus, the use of Equations (4) and (5) enables one to perform this without an introduction of a rigorous boundary between the saturation regime (grey curves) and the intermediate coverage area (orange). Such a boundary (a point between solid and dashed grey curves in Figure 1) will be imposed later.

Step 2 is related to the determination of the intermediate area with its boundaries. Technically, the most obvious way to model the intermediate region of concentrations would be to perform some interpolation. One would need to identify boundaries $x_{m}$ and $x_{s}$ of the intermediate area and to perform linkage on the class of continuously differentiable functions. Whereas boundary $x_{m}$ is naturally given as the experimental data point $\left(x_{m}\right.$, $f_{1 m}$ ) for the biggest concentration (it depends on an experimentalist's judgement whether the power law area has not yet been left there), point $x_{s}$ is to be determined on the basis of some consideration, at which $x$ the saturation regime begins. This would be a relative judgement. To avoid this, one can properly determine $x_{s}$ within the method itself linking the intermediate and saturation regime areas. We employ in the intermediate area a quadratic form $f_{2}(x)$ to be continuously linked with the adsorption function $f_{1}(x)$ in the area of small and moderate coverages on the left and with adsorption function $f_{3}(x)$ in the saturation regime area on the right. Out of parabolic, hyperbolic, and elliptic forms we chose parabola whose upper branch in a Cartesian coordinate system with apostrophe has 
the canonical form $f_{2}^{\prime 2}=2 p x^{\prime}$. Accompanying the linking point arbitrary for a while with sub/superscript " 0 ", we use the expression for a tangent line to our parabola at this point:

$$
f_{2}^{\prime} f_{2}^{(0)^{\prime}}=p\left(x^{\prime}+x_{0}^{\prime}\right)
$$

where $f_{2}^{(0)^{\prime}} \equiv f_{2}{ }^{\prime}\left(x_{0}^{\prime}\right)$. In order to continuously differentiably adjust the parabola to $f_{1}(x)$ and $f_{3}(x)$ at $x_{m}$ and $x_{s}$, respectively, we apply a translational transformation $x^{\prime}=x+c$, $f_{2}^{\prime}=f_{2}+d$, where $c$ and $d$ are constants to be determined later, to Equation (6):

$$
f_{2}(x)=\frac{p}{f_{2}^{(0)}+d} x+p \frac{x_{0}+2 c}{f_{2}^{(0)}+d}-d
$$

where $f_{2}^{(0)} \equiv f_{2}\left(x_{0}\right)$. At $x_{0}=x_{m}$, the tangent line (7) is expected to coincide with $f_{1}(x)^{\prime}$ s tangent line. As the latter has the form

$$
f_{1}(x)=K \alpha x_{m}^{\alpha-1} x+K x_{m}^{\alpha}(1-\alpha)
$$

the continuously differentiable linkage conditions at $x_{0}=x_{m}$ instantly follow:

$$
\begin{gathered}
\frac{p}{f_{1 m}+d}=K \alpha x_{m}^{\alpha-1} \\
p \frac{x_{m}+2 c}{f_{1 m}+d}-d=K x_{m}^{\alpha}(1-\alpha)
\end{gathered}
$$

At $x_{0}=x_{s}$, adsorption function $f_{3}(x)$ produces its tangent line with the equation

$$
f_{3}(x)=\frac{b}{\left(x_{s}+b\right)^{2}} x+\frac{x_{s}^{2}}{\left(x_{s}+b\right)^{2}}
$$

from where and according to Equation (7) the continuously differentiable linkage conditions at $x_{0}=x_{s}$ follow:

$$
\begin{gathered}
\frac{b}{\left(x_{s}+b\right)^{2}}=\frac{p}{f_{3 s}+d} \\
\frac{x_{s}^{2}}{\left(x_{s}+b\right)^{2}}=p \frac{x_{s}+2 c}{f_{3 s}+d}-d
\end{gathered}
$$

where $f_{3 s} \equiv f_{3}\left(x_{s}\right)$. Thus, 4 parameters $\left(c, d, p\right.$, and $\left.x_{s}\right)$ appear to be determined through four equations at the linkage points at $x_{m}$ and $x_{s}$, that is, the right linkage point itself has turned out to be determined within this procedure. The solution of set of Equation (8) can be represented in the analytical form of relations between parameters $c, d$, and $p$.

$$
\begin{gathered}
p=\alpha \frac{f_{1 m}}{x_{m}}\left(f_{1 m}+d\right) \\
d=f_{1 m}\left[2 \alpha\left(\frac{c}{x_{m}}+1\right)-1\right] \\
c=\frac{1}{2} \frac{x_{s}^{2}-\left(x_{s}+b\right)^{2} f_{1 m}(1-2 \alpha)-x_{s} b}{b-\alpha \frac{f_{1 m}}{x_{m}}\left(x_{s}+b\right)^{2}}
\end{gathered}
$$

with a transcendental equation for $x_{s}$ 


$$
\alpha f_{1 m}\left[\alpha f_{1 m}-\frac{x_{m} b}{\left(x_{s}+b\right)^{2}}\right] \frac{x_{s}^{2}-\left(x_{s}+b\right)^{2} f_{1 m}(1-2 \alpha)-x_{s} b}{x_{m} b-\alpha f_{1 m}\left(x_{s}+b\right)^{2}}+2 \alpha^{2} f_{1 m}^{2}=\frac{x_{m} b}{\left(x_{s}+b\right)^{2}}\left[\frac{x_{s}}{x_{s}+b}-f_{1 m}(1-2 \alpha)\right]
$$

which has a well localised sought root in the area $x \gg x_{m}$ and is to be easily solved numerically. As it follows from the parabola canonical form above with the translational transformation applied, the equation for the parabola bridging the chasm in the intermediate area $x \in\left(x_{m}, x_{s}\right)$ has the form:

$$
f_{2}(x)=\sqrt{2 p(x+c)}-d
$$

presented in Figure 1 in orange.
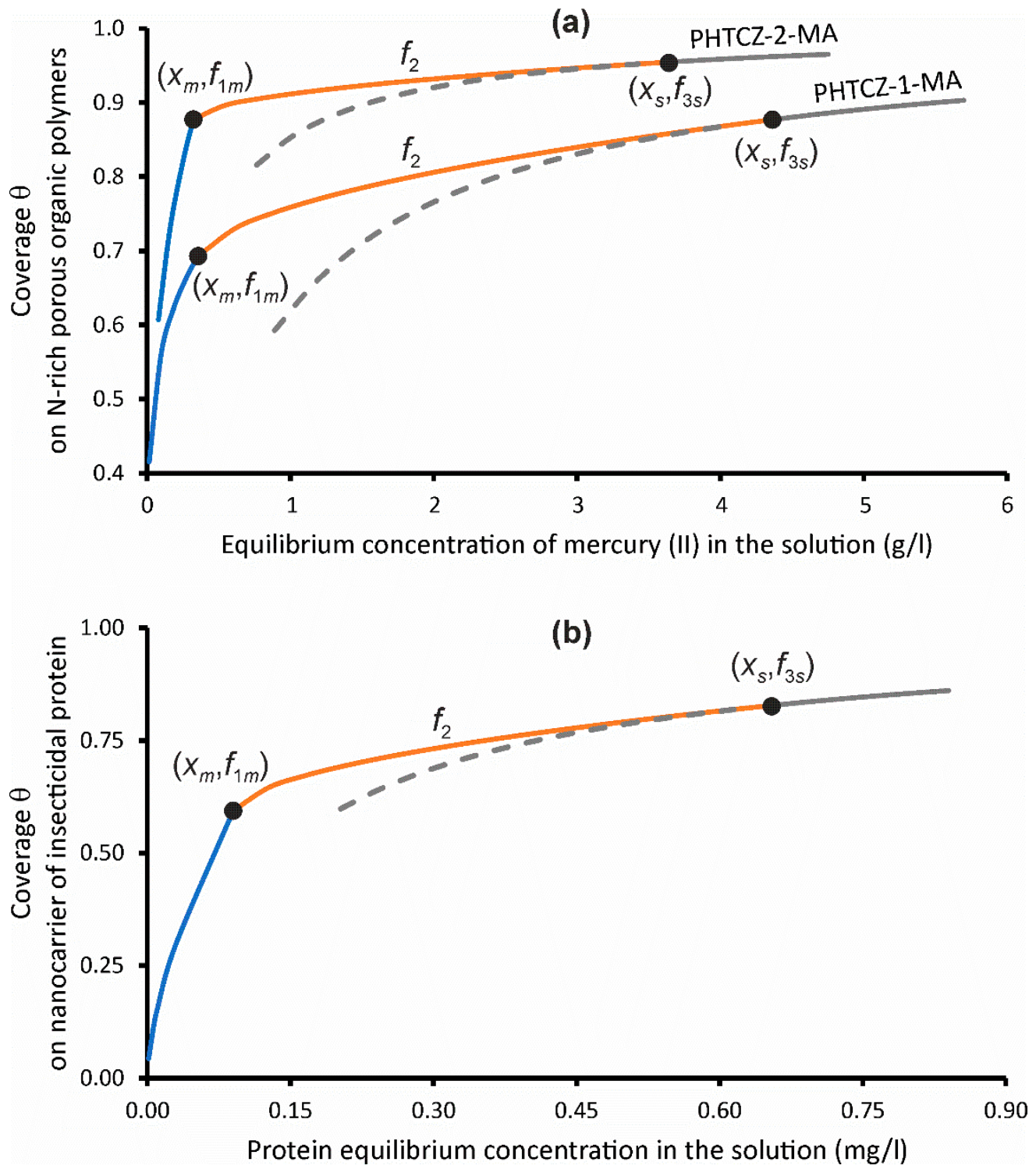

Figure 1. Freundlich isotherms in all concentration ranges. Blue $f_{1}(x)$ : experimental data (a) [6]: the source graphical data were digitalised for further analysis; (b) [9]: available supplementary digital data were used). Grey $f_{3}(x)$ : saturation regimes according to Equation (4). Orange $f_{2}(x)$ : bridging the chasm for intermediate concentrations. 
As in step 3, one has to check if the bridging between the low and high concentration regions has been performed correctly. The first and obvious condition is to be checked is certainly

$$
x_{s}>x_{m}
$$

Further obligatory conditions are

$$
f_{3}\left(x_{s}\right)>f_{1}\left(x_{m}\right), d f_{3}\left(x_{s}\right) / d x<d f_{1}\left(x_{m}\right) / d x
$$

otherwise, geometrically obviously, the bridging would not be possible. If conditions (11) are not met, either the approximation of the experimental data with Equation (1) went beyond the region in which the power law is indeed valid, or the Freundlich AM is not the true one for the system considered.

\section{Results and Discussion}

The parameters finally determined for the systems considered in Figure 1 are presented in Table S1.

The calculations performed demonstrate the most important practical result indicating how close to (or far from) the saturation regime the rightmost experimentally determined point is situated. Alongside this de facto extension of the source works, values $b=b_{0} /\left(1+\frac{1}{\alpha}\right)$ in Equation (4) appear to be determined and to characterise both saturation rate and surface adsorption properties at big coverages. How properly the chasm in the intermediate area has been bridged, is to be judged on the basis of experimental data to be obtained for the corresponding region. Even in the framework of the proposed approach, one could choose between at least three global linkage options, that is, parabolic, hyperbolic, and elliptical ones with a lot of opportunities (for example, to add the rotational transformation) for local tuning.

The concentration ranges studied experimentally in the works considered above appeared to be easily analytically continued (in the intermediate area approximately) from $0.09 \mathrm{mg} / \mathrm{L}$ (protein on nano- $\mathrm{Mg}(\mathrm{OH})_{2}$ ) and $300 \mathrm{mg} / \mathrm{L}$ (mercury (II) on N-rich porous organic polymers) up to "reasonable infinity".

\section{Conclusions}

In view of umpteen works having employed the Freundlich isotherm for interpretation of experimental data, Zeldowitch's theory of the corresponding AM has been revisited to demonstrate an analytical continuation from typically scant data sets for small and moderate coverages to the area of saturation regime. This enables one to judge the saturation rate and to bridge a chasm between low (moderate) and high pressure (activity or concentration) areas. Experimental data for adsorption of mercury (II) on N-rich porous organic polymers and of protein on nano- $\mathrm{Mg}(\mathrm{OH})_{2}$ published by independent researchers have been processed to demonstrate how the analytical continuation method of the Freundlich isotherm developed in this article works.

J. Zeldowitch underlined in his historical work that his goal had been to contribute to the correct interpretation of the community's experimental data rather than to develop a quantitative theory of adsorption on heterogeneous surfaces [12,13]. Like that, we are to emphasize our goal to be to suggest a framework useful for analysis of the community's experimental data first recognised as complying with the Freundlich isotherm but indeed as those to be essentially more comprehensively interpreted within this framework of Freundlich-Zeldowitch AM.

Supplementary Materials: The following are available online at https:/ / www.mdpi.com/article/ 10.3390 /app11178078/s1, Table S1: Parameters of the systems with adsorbate and adsorbent (the isotherms are presented in the article's Figure 1).

Author Contributions: Conceptualization, M.V.; methodology, M.V.; validation, L.T. and M.V.; formal analysis, M.V.; resources, A.P.; data curation, E.T.; writing—original draft preparation, M.V.; 
writing-review and editing, M.V.; supervision, L.T.; project administration, A.P. All authors have read and agreed to the published version of the manuscript.

Funding: This research received no external funding.

Institutional Review Board Statement: Not applicable.

Informed Consent Statement: Not applicable.

Data Availability Statement: Not applicable.

Conflicts of Interest: The authors declare no conflict of interest.

\section{References}

1. Vigdorowitsch, M.; Tsygankova, L.; Prokhorenkov, V. The polylogarithmic adsorption function: A linear energetic surface heterogeneity. J. Math. Chem. 2020, 58, 1353-1363. [CrossRef]

2. Vigdorowitsch, M.; Tsygankova, L.E.; Tanygina, E.D. Approximate analytical overall isotherms: Lateral interactions of adsorbate species and patchwise topology. Heliyon 2020, 6, e05738. [CrossRef] [PubMed]

3. Redlich, O.; Peterson, D.L. A Useful Adsorption Isotherm. J. Phys. Chem. 1959, 63, 1024. [CrossRef]

4. Liu, H.; Xu, G.; Li, G. Preparation of porous biochar based on pharmaceutical sludge activated by $\mathrm{NaOH}$ and its application in the adsorption of tetracycline. J. Colloid Interface Sci. 2021, 587, 271-278. [CrossRef] [PubMed]

5. $\quad$ Rodrigues, S.S.M.; Ribeiro, D.S.M.; Frigerio, C.; Costa, S.P.F.; Prior, J.A.V.; Pinto, P.C.A.G.; Santos, J.L.M.; Saraiva, M.L.M.F.S.; Passos, M.L.C. Immobilization of Distinctly Capped CdTe Quantum Dots onto Porous Aminated Solid Supports. ChemPhysChem 2015, 16, 1880-1888. [CrossRef] [PubMed]

6. Sang, Y.; Cao, Y.; Wang, L.; Yan, W.; Chen, T.; Huang, J.; Liu, Y.-N. N-rich porous organic polymers based on Schiff base reaction for $\mathrm{CO}_{2}$ capture and mercury(II) adsorption. J. Colloid Interface Sci. 2021, 587, 121-130. [CrossRef] [PubMed]

7. Alghamdi, A.A.; Al-Odayni, A.-B.; Saeed, W.S.; Al-Kahtaniet, A.; Alharthi, F.A.; Aouak, T. Efficient Adsorption of Lead (II) from Aqueous Phase Solutions Using Polypyrrole-Based Activated Carbon. Materials 2019, 12, 2020. [CrossRef] [PubMed]

8. Edet, U.A.; Ifelebuegu, A.O. Kinetics, Isotherms, and Thermodynamic Modeling of the Adsorption of Phosphates from Model Wastewater Using Recycled Brick Waste. Processes 2020, 8, 665. [CrossRef]

9. Pan, X.; Xu, Z.; Zheng, Y.; Huang, T.; Li, L.; Chen, Z.; Rao, W.; Chen, S.; Hong, X.; Guan, X. The adsorption features between insecticidal crystal protein and nano- $\mathrm{Mg}(\mathrm{OH})_{2}$. R. Soc. Open Sci. 2017, 4, 170883. [CrossRef] [PubMed]

10. Romero, J.R.G.; Moreno-Piraján, J.C.; Gutierrez, L.G. Kinetic and Equilibrium Study of the Adsorption of $\mathrm{CO}_{2}$ in Ultramicropores of Resorcinol-Formaldehyde Aerogels Obtained in Acidic and Basic Medium. J. Carbon Res. 2018, 4, 52. [CrossRef]

11. McBain, J.W. The Sorption of Gases and Vapors on Solids, 1st ed.; Geo Routledge and Sons: London, UK, 1932; 577p.

12. Zeldovich, Y.B. Selected Works of Yakov Borisovich Zeldovich, Volume 1: Chemical Physics and Hydrodynamics, 1st ed.; Princeton Legacy Library: Princeton, NJ, USA, 2014.

13. Zeldowitch, J. Adsorption site energy distribution. Acta Physicochim. URSS 1934, 1, 961-973.

14. Rudnitsky, L.A.; Alexeyev, A.M. Equations of adsorption isotherms for heterogeneous surfaces. J. Catal. 1975, 37, 232-239. [CrossRef]

15. Rudnitsky, L.A.; Alexeyev, A.M. The equation of adsorption isotherm with exponential distribution of centres on adsorption heat. Doklady AN SSSR 1972, 205, 1169-1172.

16. Thomas, J.M.; Thomas, W.J. Introduction to the Principles of Heterogeneous Catalysis; Academic Press: New York, NY, USA, 1967; 544p. 